\title{
Modelling and simulation of controlled depth abrasive water jet machining (AWJM) for roughing passes of free-form surfaces
}

\author{
Yigit Ozcan ${ }^{1} \cdot$ Lutfi Taner Tunc $^{1}$ (D) $\cdot$ Jan Kopacka ${ }^{2} \cdot$ Beril Cetin $^{1} \cdot$ Matej Sulitka $^{2}$ \\ Received: 5 May 2020 / Accepted: 19 April 2021 / Published online: 28 April 2021 \\ (C) The Author(s), under exclusive licence to Springer-Verlag London Ltd., part of Springer Nature 2021
}

\begin{abstract}
Controlled depth abrasive waterjet machining (AWJM) is an unconventional and promising process for materials introducing challenges in conventional machining for high value manufacturing (HVM) industries such as aerospace and automotive. In such applications, waterjet acts as a flexible cutting tool compared to conventional milling. The kerf profile, i.e. the removed material volume, nonlinearly depends on the process parameters such as water pressure, jet traverse speed, abrasive rate, and stand-offdistance unlike the deterministic case in mechanical milling. Thus, prediction of kerf profile, i.e. width, depth, and shape, is of great importance for accurate and efficient process development and tool path generation in AWJM. In this paper, a novel analytical model is proposed for prediction of kerf profile, relying on conservation of energy and momentum, where the material removal is related to the machinability number of the workpiece material, so that the requirement of calibration tests is eliminated. Then, the proposed AWJM process model is used in a framework to predict 3D in-process workpiece (IPW) geometry, which is represented using signed distance field (SDF) approach. The process model is verified by experimental results with an average error of $15 \%$, where the machine profiles are measured by confocal optical microscopy. In some of the experiments, undulated material removal was observed which is due to inconsistent abrasive supply of the system, which is considered to be the major source of the kerf depth prediction errors. The IPW simulation model is verified by variable jet traverse speed AWJM experiments, considering the significant effect of jet traverse speed on the kerf depth. The machined specimens are sectioned along the jet traverse direction and the cross section of the test pieces is visually compared with the simulations. The machined profiles introduced some amount of undulated profile, which may be attributed to the non-consistent abrasive supply in the system. It is shown that the proposed IPW simulation approach demonstrates a reasonable accuracy to plan controlled depth AWJM processes.
\end{abstract}

Keywords Waterjet Machining $\cdot$ Workpiece $\cdot$ Simulation

\section{Introduction}

Together with the development of alternative material removal processes [1], hybrid machining strategies are being evolved, as well [2]. Machinability and tool life in conventional milling of composites, $\mathrm{Ni}$, and $\mathrm{Ti}$ alloys are low. Additionally, very soft materials are destroyed in conventional

Lutfi Taner Tunc

ttunc@sabanciuniv.edu

1 Integrated Manufacturing Research and Application Centre, Faculty of Engineering and Natural Sciences, Sabanci University, Istanbul, Turkey

2 Faculty of Mechanical Engineering, Czech Technical University, Horská 3, 12800 Praha 2, Czech Republic milling. Thus, AWJM is advantageous for such materials introducing challenges in conventional machining $[3,4]$. In industry, one use of AWJM surface modification [5]. For machining purposes, it is mostly used for $2 \mathrm{D}$, through thickness cutting. However, recently controlled depth use has been proposed to replace challenging roughing passes to achieve crack-free [6] and smooth surfaces [7]. Another application of abrasive material removal can be mentioned as abrasive flow machining (AFM), which caught the attention of the researchers in this area. Wei et al. [8] proposed a new material removal model to predict the material removal distribution along the flow direction, which helped controllability of the process.

In AWJM, potential energy of the pressurized water is converted to kinetic energy as the water and abrasive particle velocity increase throughout the travel along the orifice. To 
achieve increased cutting efficiency for titanium and nickel alloys, which are known with their low machinability, high velocity water is mixed with small abrasive particles. AWJM, while providing a machining capability for such metals, stands as an alternative machining method for deep cutting of relatively softer materials such as steel and aluminium. Aerospace parts such as centre wing box, wing skins, vertical stabilizer, stiff rotors, and turbine blades are industrial parts that can be considered the typical parts benefiting from AWJM.

Momber and Kovacevic [9] emphasized that minimized burr, reduced work hardening, higher fatigue life, and machining capability for thick and hard materials are the other important advantages of AWJM. They also discussed different techniques for utilization of AWJM, where controlled depth machining was mentioned as one of the most challenging alternatives among its versatile use. Similarly, in a recent CIRP keynote paper on high energy fluid jet machining, Axinte et al. [4] emphasized that AWJM eliminates the tool wear issue especially in machining of hard materials. This was elaborated as an advantage of AWJM in cutting for hard materials such as titanium and nickel, used in aerospace industry.

In the literature, there have been important studies focusing on the effect of process parameters on the process performance. Fowler et al. [10] investigated the controlled depth AWJM of Ti6Al4V, which is a common material used in aerospace industry. They experimentally showed that the traverse speed governs the process in terms of surface waviness and material removal rate. In their investigation, they concluded that waviness increases with increased number of passes. It was also concluded that as the grit size decreases, the material removal rate, waviness, and roughness decreases. However, as being an experimental work, its application and the validity of the results are limited to the experimental condition. In another study, Azmir and Ahsan [11] experimentally studied the generation of tapered kerf geometry in AWJM of glass/ epoxy composite laminates, which was considered one of the major geometrical inaccuracy sources. They primarily used Taguchi method to identify experimental conditions and used ANOVA techniques to identify the effect of process parameter on surface roughness and kerf taper. They identified that hydraulic pressure and abrasive type govern surface roughness and kerf taper. However, this study did not propose a modelling approach. Löschner et al. [12] studied the effect of jet traverse speed on surface quality in AWJM of 10-mm thick $316 \mathrm{~L}$ stainless steel. In their experimental study, they demonstrated that jet traverse speed significantly affects surface roughness, surface quality, and machining marks especially at the deeper sections of the kerf. They showed the trade-off between jet cutting speed and the surface quality in AWJM, as well. In another recent experimental study, Shukla et al. [13], experimentally studied the effect of AWJM parameters based on Taguchi and Evolutionary optimization techniques. Jet transverse speed, standoff distance, and mass flow rate were considered the effective parameters included in the parameter study in order to investigate their effects on kerf top width and taper angle. They developed regression models, where the experimental data was correlated to the process parameters. Advanced optimization techniques such as particle swarm optimization, firefly algorithm, artificial bee colony, simulated annealing, black hole, biogeography based, and nondominated sorting genetic algorithm were implemented for parameter selection in AWJM. They concluded that biogeography algorithm shown to be performing much better compared to others. Uthayakamur et al. [14] experimentally studied machinability of Ni-based super alloys, a common material for aerospace, by AWJM. They focused on process parameters such as jet pressure, traverse speed, and stand-off distance at three levels. They measured the geometrical parameters making up the kerf, such as width, taper, and material removal rate. They used SEM images to evaluate the surface morphology and material removal mechanism. One of the important conclusions was the significant effect of water jet pressure on the kerf geometry and surface quality. Wang et al. [15] investigated the kerf geometry in AWJM focusing on the effect of process parameters on the kerf profile. They proposed an approach based on curve fitting to experimentally measured kerf profiles, and then demonstrated that the fitted, i.e. calibrated, function can be used to predict the AWJM kerf geometry. However, this approach requires several testing efforts to perform curve fitting. Yuvaraj et al. [16] performed an experimental study on investigating the effect of process parameters on AWJM of D2 steel. The experimental results were analysed through ANOVA analysis. As a result, jet pressure of $225 \mathrm{MPa}$, abrasive mesh size of \#100, and jet impingement angle of $70^{\circ}$ were identified to provide improved part quality for D2 steel. The experimental efforts were significantly beneficial to understand the AWJM process under various conditions, though process planning and improvement require predictive models.

Since the introduction of AWJM, modelling of the kerf profile has been another interesting focus for researchers. In one of the early efforts on modelling, Finnie [17] studied several aspects of AWJM such as the fluid flow conditions for erosion, and material removal mechanism for ductile and brittle materials. They developed an erosion model for single abrasive particle in the fluidic medium, which performed well to predict the erosion mechanism for ductile materials. They defined the erosion problem by two phenomena, (i) particle motion in fluidic environment and (ii) the response of the part surface during particle struck. They concluded that surface roughness increases on the contact area and accelerates the erosion rate at the presence of fluid turbulence. Bitter [18] [19] developed a model for wear and deformation to explain abrasive mechanism in slurry transport at high fluid jet. The proposed model worked fine at high impact angles, where particles are assumed as ideal spheres and repeated impact 
takes place on the surface, causing elastic and plastic deformation, where strain hardening effect due to deformation is neglected. As the abrasive particles are assumed to cause elastic impact, the workpiece material deformation is modelled as elastic-plastic, relying on energy conservation phenomena. The model also considers the particle pull out effect on the workpiece material at the instant of collision. It is assumed that the volume of the material removed from part surface and plastic deformation energy are proportional. That proportionality is satisfied by a deformation wear factor. The deformation and wear mechanism are considered to predict the overall material removal. However, this model requires wear factor measurements at various abrasive particle velocity values. Hashish and Du Plessis [20] proposed a material removal model as extension to the studies of Finnie [17] and Bitter $[18,19]$. They neglected through-thickness abrasive particle velocity, jet spreading, and erosion due to water energy, assuming uniform distribution of the abrasive particle throughout the jet cross section. They considered that cutting wear and deformation are dominant at low and high impact angles, respectively.

In all energy conservation models, abrasive particle impact velocity needs to be known for accurate predictions as the total kinetic energy of the abrasive particles cause erosion. Tazibt et al. [21] proposed a model based on prediction of acceleration of abrasive particles and water jet, with the assumption of smoothly changing jet velocity causes the abrasive particles to accelerate along the standoff distance. Water jet and abrasive particle velocities are well correlated with experimental results using mass and momentum conservation. Capello and Gropetti [22] proposed another energy dissipation-based model by relating the kinetic energy of the abrasive particle to the mechanical properties of the workpiece material. Abrasive particles' kinetic energy is dissipated to remove material with increased resistance to wear, which makes model more realistic with respect to previously proposed models. They included the exposure time effect, as well. Raju et al. [23] modelled erosion depth based on the approach proposed by Hashish [20]. They assumed the existence of (i) smooth cutting and (ii) rough cutting and introduced three empirical constants, which are identified by prior calibration experiments. They found that the material flow stress is in the range of $1 / 2$ to $1 / 30$ of the elastic modulus. Friction coefficients were proven to be 10 times higher than the model proposed by Hashish [20]. Raju et al. [23] also showed that there is high amount of reduction in velocity due to wall drag, which dominantly affects the cutting performance in deep cut applications. Momber and Kovacevic [24] introduced an energy dissipation function and related the erosion depth to the energy absorption rate. They separated and calibrated the energy dissipation parameters such as damping, friction, and erosion debris generation, by performing material removal and fracture tests. One of the important developments in modelling of AWJM processes was proposed by Hoogstrate et al. [25] as they related the machinability number and specific cutting energy to predict material removal. EITobgy et al. [26] covered curved surfaces in a modified the erosion model by Finnie et al. [17], where another step ahead was being able to simulate multiple particle erosion, using the standard properties of workpiece material. Pi and Tuan [27] predicted the required cutting energy by using physicalmathematical models and experimental methods using Buckingham-Pi theorem. They were able to predict the effects of AWJM parameters such as abrasive size, abrasive material, and workpiece material on the process performance. In a recent work, Ahmed et al. [28] numerically studied the effect of particle impact on the cut surface, i.e. the kerf wall. They showed that the abrasive particle impact angle depends on the radius of curvature of the generated kerf geometry. The abrasive particles shown that they tend to slide or stay around the cutting surface when the radius of curvature is larger at low impact angles. One of the important results of their study was the variation of particle impact velocity among the cutting regions. They concluded that the velocity of the particles significantly decreases due to fluid drag.

In prediction of kerf geometry, calibration-based approaches were used in the literature, as well. Axinte et al. [29] related the erosion rate to the particle velocity in the form of an exponential function such as $E(r)=C(V n)^{k}$, where $C$ and $k$ are material positive constants, $\mathrm{V}$ is particle velocity and, $\mathrm{n}$ is particle impact direction. This approach explains that the kerf profile erodes in particle direction and the kerf depth is proportional to the particle velocity, which requires geometrical measurement of the kerf profiles for pre-defined cases used for calibration. Billingham et al. [30] proposed calculation of the dimensionless erosion rate to predict kerf footprint at different feed rates. Although Axinte et al. [29] and Billingham et al. [30] cover a wide range of jet traverse velocities, the prediction error increases at lower jet feed velocities, where erosion rate is high. Axinte et al. [29] addressed such issues by introducing a correction factor to the model, which improved the prediction accuracy. In one of the recent studies on modelling of kerf geometry, Gilles et al. [31] proposed a new cutting depth model which requires a rapid calibration for cutting of open rectangular pockets with implementation on Ti-6Al-4V. The calibration phase included elementary passes and three pocket machining passes at the required machine configuration, pressure level, and abrasive flow rate. Kerf profile was assumed to be in the form of a Gaussian Curve, which was fitted based on the jet traverse speed. The calibration experiments were used to identify the coefficients in the Gaussian function. In the experimental verification, they achieved accuracy levels of 5\%. However, considering that the calibrated coefficients are valid for the calibrated parameter range, this would limit the application of the model for parameter selection. In addition, the requirement of calibration tests may significantly increase the effort for process development.

In addition to analytical approaches, there are also numerical methods to predict kerf profile. Anwar et al. [32] 
developed a finite element (FE) analysis based automatic simulation of the eroded surface in the case of overlapping jet footprints. They targeted a range of step-over distances, water pressure, and jet traverse velocity. They achieved $15 \%$ of error margin according to the verification experiments performed on Ti6Al4V, where garnet abrasives were used. Kumar and Shukla [33] presented elasto-plastic-based FE model to predict the erosion in AWJM. They were successful in modelling the effect of multi-particle impact, where they demonstrated crater shape of the eroded volume in terms of spherical shape and depth.

Although virtual machining [34] is well established for conventional milling, turning, and grinding, there is yet to be an approach to simulate the IPW in AWJM. Thus, benefits of the process modelling efforts may not be well reflected to the industry. As the IPW continuously varies, fast and accurate simulation of material removal requires appropriate techniques to be integrated with AWJM process models. Compared to the alternative methods such as constructive solid geometry and boundary representation, signed distance field [35-37] stands by far more efficient in describing moving boundaries.

This paper aims to contribute to AWJM literature by developing (i) a generalized, calibration-free process model and (ii) an integrated simulation scheme to predict IPW geometry in controlled depth AWJM used. Along these aims, a method for prediction of kerf profile is proposed based on the material machinability number and abrasive velocity. The major contribution of this approach is the elimination of prior experimental calibration procedures for prediction. The proposed method is verified by experimental results both from literature and new cutting tests. To validate the generality of the proposed model in prediction of the kerf profile, cutting tests are performed at various pressures, feed rates, and abrasive flow rate conditions. Reasonable accuracy is obtained at an average error of $15 \%$, where minimum of $1 \%$ and maximum of $30 \%$ absolute error was achieved in the cutting experiments. Model gives accurate results for prediction of kerf depth in abrasive waterjet machining of various materials as compared with the data in the literature. Henceforth, the manuscript is organized as follows; Section 2 presents the proposed model for kerf prediction, which is followed by the signed-distance field (SDF)-based approach in simulation of AWJM machining cycles. In Section 4, experimental verification and discussion of the experiments are presented. The study is finalized by conclusions in Section 5.

\section{Modelling of AWJM}

Controlled depth AWJM is an alternative to conventional milling in roughing passes, where dimensional accuracy and surface roughness requirements are loose. However, it is important to know the kerf depth for planning of multi-pass roughing cycles. Considering the unconventional nature of AWJM, which demonstrates flexible and parameterdependent cutting envelope, it is required to predict the kerf geometry in controlled depth AWJM.

In this section, the proposed process model, for kerf prediction in AWJM, is explained. In Section 2.1, process parameters are described. This is followed by the energy equation in relation to volumetric calculation approach and prediction of material removal together with the energy heads, in Section 2.2. The pump pressure is related to the water jet flow through energy conversation. The kinetic energy transfer from the water jet to the abrasive particles is established through the momentum conversation [21]. Finally, the material removal is related to the kinetic energy of the abrasive particles and the specific cutting energy of the material, which is extracted from the machinability number of the material, available in the literature. The major benefit of the proposed approach, compared to the models in the literature, is elimination of any need for calibration tests.

\section{AWJM process parameters and geometry}

In the proposed process model, the effect of pump pressure, abrasive flow rate, feed rate, standoff distance, nozzle size, abrasive size, orifice size, and workpiece material (see Fig. 1a and Fig. 1b) is taken into account. The high pressure water enters the nozzle at point 1 (see Fig. 1b), the abrasives are mixed with the water jet flow at point 2 , and then the high velocity abrasive water jet exits the nozzle at point 3 to the atmospheric pressure. The kerf geometry is expressed in terms of kerf width and depth, where the shape is assumed to rely on Gaussian shape as shown in Fig. 1c. Due to nature of fluid flow, the jet velocity varies along the radial direction (see Fig. 1d), which is also considered in the process model.

In the literature, the studies show that the kerf depth is inversely proportional with feed rate and directly proportional with abrasive flow rate and pump pressure. The other parameters such as stand-off distance and abrasive size may have nonlinear effects on the kerf profile. Following the definition of process geometry and parameters in AWJM, the next section explains the details of the energy-based kerf geometry prediction.

\subsection{Energy-based modelling of kerf profile}

The proposed model involves the steps given in Fig. 2, which take the process parameters as input and then pass through conservation of momentum and mass to predict kerf depth. Pump pressure, $P_{w}$, and water density is used to derive water jet velocity by Bernoulli equation. Abrasive flow rate, $\dot{m}_{a}$, and 
Fig. 1 Abrasive waterjet machining process parameters. (a) Geometrical parameters, (b) Process parameters, (c) Kerf geometry, (d) Jet velocity profile

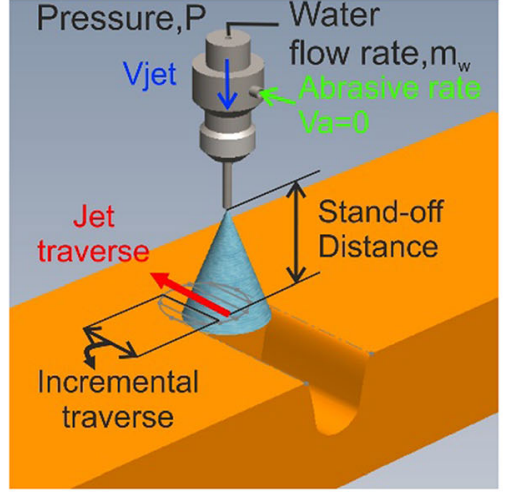

(a)

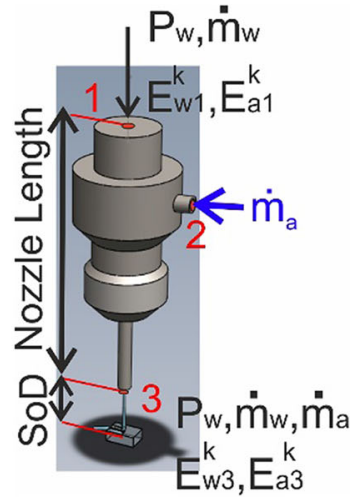

(b)

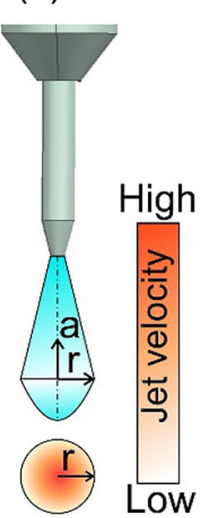

(d)

(c)

energy of the splashed abrasives and water jet. Then, Bernoulli Equation is written for the energy balance at the inlet and exit of the nozzle, as follows:

$E_{a, 4}^{k}+E_{w, 4}^{k}=e_{w p} V_{\mathrm{kerf}}+E_{a, 5}^{k}+E_{w, 5}^{k}$

where $K E_{p, 4}$ is the kinetic energy of abrasives just before impact, $K E_{w, 4}$ is the specific cutting energy of the workpiece before impact, $e_{w p}$ is the specific cutting of the workpiece energy required to eroded volume of material, the kinetic

water flow rate, nozzle length, abrasive and nozzle diameter, pansion angle, abrasive and nozzle diameter, standoff distance, mass of the abrasive particle, and abrasive flow rate.

Theoretically, the total kinetic energy of the abrasive particles and the water jet at the nozzle exit are equated to the

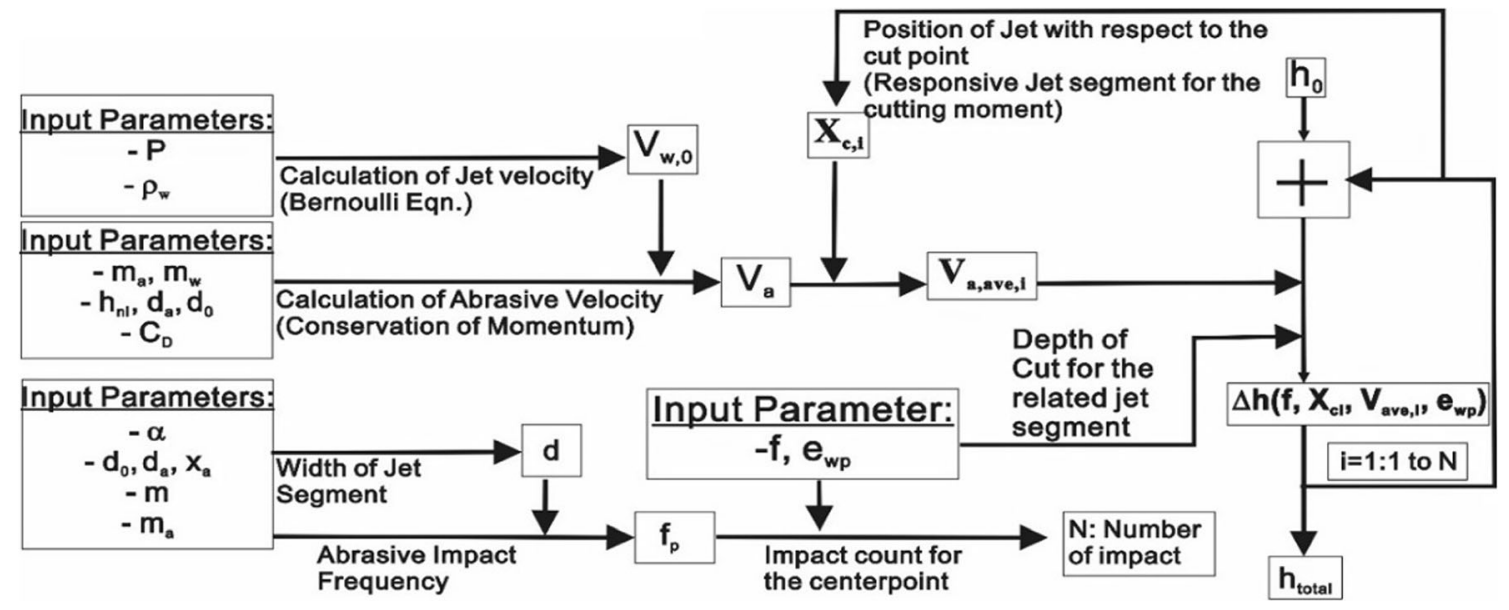

Fig. 2 Proposed approach 
material, $V_{\text {kerf }}$ is the kerf volume, $K E_{p, 5}$ is the kinetic energy of abrasives just after impact, and $K E_{w, 5}$ is the kinetic energy of water jet just after impact

The energy balance given in Eq. (1) is simplified to the following form under two fundamental assumptions.

$E_{a, 4}^{k}=e_{w p} V_{k e r f}$

Assumption 1: - Erosion is due only the abrasive particle energy [25]. Thus, the kinetic energy of the water jet just before impact, $E_{w, 4}^{k}$, and just after impact, $E_{w, 5}^{k}$, is assumed to be equal, which is verified by pure water jet machining experiments as presented in Section 4.2.

$E_{w, 4}^{k}=E_{w, 5}^{k}$

Assumption 2: - As abrasive particles embed or accumulate, at almost zero velocity, on the part surface it is assumed that all the abrasive energy is spent for erosion. Kinetic energy of the abrasives after impact, $E_{a, 5}^{k}$, is assumed to be zero.

$E_{a, 5}^{k} \simeq 0$

Considering the variation of the abrasive velocity in the radial direction (see Fig. 1d), the jet area is divided into radial segments to account the effect of velocity profile. The material removal of each segment is recursively accumulated throughout the jet traverse to predict the kerf profile based on the approach given in Fig. 2.

The kinetic energy of abrasive particle is written in terms of average abrasive velocity and mass, and then putting into Eq. (2):

$\frac{1}{2} M_{a} V_{a v e, 1}^{2}=e_{w p} V_{k e r f}$ and $M_{a}=\dot{m}_{a} \frac{1}{f}$

Volume of the infinitesimal material removal is written as:

$\Delta V_{\text {kerf }}=d l X_{c} \Delta h$

where $X_{c}$ and $\Delta h$ are the width and depth of the kerf, respectively. Hence, the depth, $\Delta h$, of infinitesimally removed volume is written as:
$\frac{\Delta h=\frac{1}{2} \dot{m}_{a, 1} V_{a v e, 1}^{2}}{f X_{c} e_{w p}}$

\subsubsection{Material-specific energy}

In this study, the specific cutting energy of the material, $e_{w p}$, is calculated based on the machinability number, $\mathrm{N}_{\mathrm{m}}$, of the corresponding material. In this regard, the data provided by Hoogstrate et al. [25] is used. They established an empirical relation between machinability number and specific cutting energy for various materials as plotted in Fig. 3.

In Fig. 3, it is seen that the specific cutting energy varies linearly with the machinability number, $\mathrm{N}_{\mathrm{m}}$, which can be used to establish a linear relation as below:

$e_{w p}=\frac{6.11 * 10^{11}}{N_{m}}$

\subsubsection{Calculation of abrasive jet velocity}

In this study, the velocity of the abrasive particle is predicted considering the standoff distance. The abrasive particle velocity converges to jet velocity throughout the travel of the abrasive particle in the jet [21], so that, as the nozzle length and standoff distance increases, the abrasive particle velocity converges to the water jet velocity. After reaching fully developed water jet velocity, abrasive particle velocity saturates and does not further increase significantly. Since focusing tube length is long enough to achieve fully developed jet velocity, it can be assumed that the jet velocity after the abrasive particle passes from nozzle tip does not change along a very short standoff distance, i.e. 0 to $5 \mathrm{~mm}$. Tazibt et al. [21] experimentally verified this assumption. By taking linear interpolation of velocity equation with respect to standoff distance, the abrasive particle velocity can be predicted during erosion. By using Bernoulli equation, the velocity of water jet is approximated as follows:

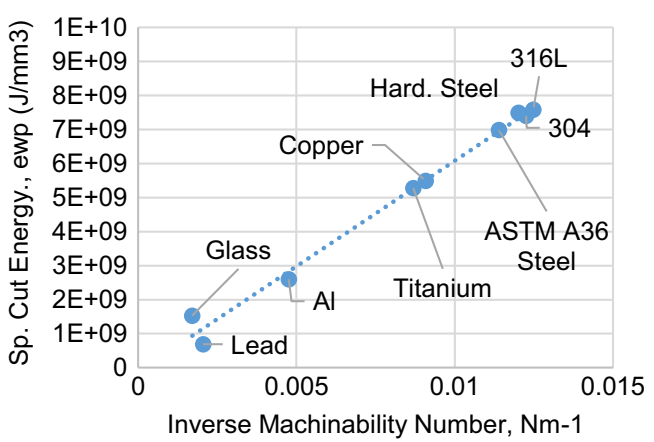

Fig. 3 Inverse machinability number vs specific cutting energy [25] 
$V_{w, 0}=\psi \sqrt{\frac{2 \mathrm{P}}{\rho_{w}}}$

where $V_{w, 0}, \psi, \mathrm{P}$, and $\rho_{w}$ are jet velocity, compressibility coefficient, mean relative water pressure, and density of water at $\Delta p$, respectively. The compressibility coefficient is written as follows:

$\psi=\sqrt{\frac{L}{\mathrm{P}(1-n)} \times\left[\left(1+\frac{\mathrm{P}}{L}\right)^{1-n}-1\right]}$

In equation (10), $\mathrm{L}$ is the reference pressure equals, i.e. 300 $\mathrm{MPa}$, and $n$ is 0.1368 at $25^{\circ} \mathrm{C}$ [38].

As modelled by Tazibt et al. [21], $m$ is the single particle mass, $\rho_{w}$ is water jet density, $\Omega_{a}$ is cross sectional area of the particle, and $C_{d}$ is drag coefficient of particle in the jet, which can be taken as 0.2 [21]. Based on long tube assumption, the equivalent velocity that both abrasive and water converges and hence the equalized velocity, Veq, is written as follows:

$V_{e q}=\frac{m_{a} V_{a, 0}+m_{w} V_{w, 0}}{m_{a}+m_{w}}$

where indices " 0 " and " 1 " represent inlet and exit points of the nozzle, respectively. As the velocity of abrasive particle at mixing chamber in the nozzle is very low, $V_{a, 0}$ is assumed to be zero [21].

In the AWJM pump system, the water flow rate, $\dot{m}_{w, 1}$, depends on the pump pressure and orifice size. In this regard, the correct flow rate value needs to be used for accurate prediction of the jet and abrasive velocity calculations. At higher pump pressures and at larger orifice diameters, the water flow rate increases. Therefore, the variation of water flow rate with pump pressure and orifice size is obtained from the pump manufacturer as shown in Fig. 4.

\subsubsection{Calculation of mass and cross-sectional area of abrasive particles}

The mass, $m$, and cross sectional area, $\Omega_{a}$, (see Fig. 5) of a single abrasive particle is approximated using the correlation between the mesh number and the mesh size of abrasives [9]. As the abrasive particles are assumed to be in spherical shape, the cross-sectional area of a single abrasive particle is written in terms of the mesh number as follows:

$\Omega_{a}=\pi \frac{\left(17.479 \times(\text { Mesh\# })^{-1.0315}\right)^{2}}{4}$

Then, the mass of a single abrasive particle is approximately written in terms of the volume and density of the particle.

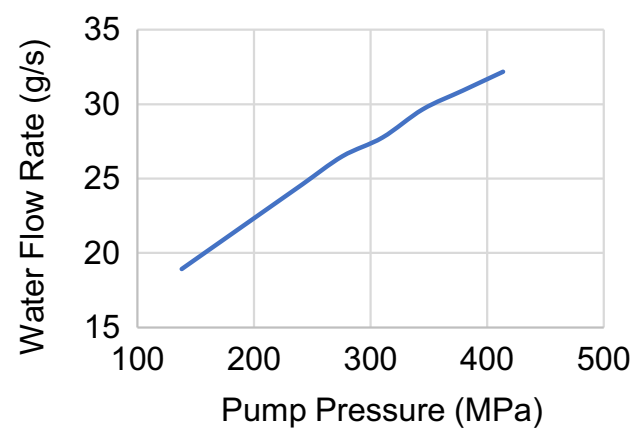

(a)

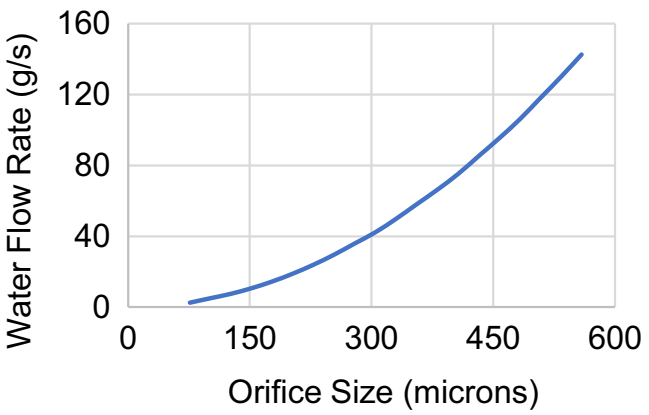

(b)

Fig. 4 Variation of water flow rate. (a) variation with pump pressure (orifice size $=254 \mu \mathrm{m}$ ) and (b) variation with orifice size (Pressure $=345$ $\mathrm{MPa})$

$m_{a}=\frac{4}{3} \rho_{a} \pi \frac{\left(17.479(\text { Mesh\# })^{-1.0315}\right)^{3}}{8}$

In order to predict the resultant penetration depth, the energy balance equation (1) needs to be solved through the exposure time of the workpiece material. Considering that erosion occurs as the particle impacts on workpiece material, it can be performed based on the particle impact frequency, $f_{p}$, at a specific point:

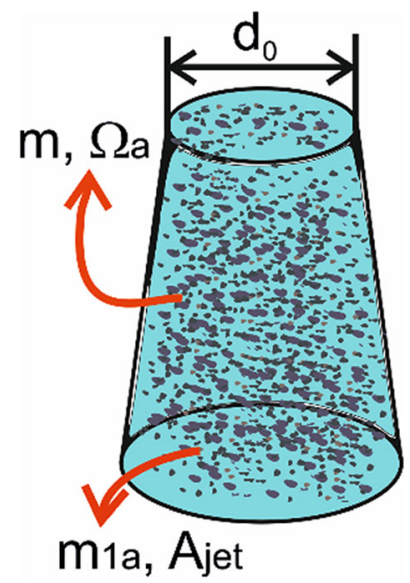

Fig. 5 Demonstration of abrasive flow in the jet 


$$
\begin{aligned}
f_{p} & =\frac{\dot{m}_{a, 1}}{m} \frac{d_{a}}{d_{j e t}}=\frac{\dot{m}_{a, 1}}{m} \frac{d_{a}}{d} \\
& =\frac{6}{\pi} \frac{\dot{m}_{a, 1}}{\rho_{a} *\left(17.479(\text { Mesh } \#)^{-1.0315}\right)^{2}} \frac{1}{2 * x_{a} * \tan \frac{a}{2}+d_{0}}
\end{aligned}
$$

Number of impacts, $\mathrm{N}$, at a specific point is written in terms of impact frequency, length, and jet feed velocity as follows:

$N=f_{p} \frac{l(x=0)}{f}=f_{p} \frac{d}{f}$

The resultant depth of cut can be summarized by following formula:

$\sum_{i=1}^{N} \frac{1}{2} \frac{m_{a, 1} \times V_{a, 1_{i}}^{2}}{f \times X_{c_{i}} \times e_{w p}}$

where $w_{i}$ and $V_{a, 1_{i}}$ are the width and average abrasive velocity of the jet segment at the nozzle feed position with respect to the initial contact point of jet on the specific point, respectively. The representation of the algorithm in a schematic figure for the first three progressive segments is shown in Fig. 6.

\subsubsection{Prediction of kerf profile}

In controlled depth AWJM, slot like kerf geometry is generated. However, because of the process nature, the kerf profile is wider than the nozzle tip diameter, concave at the top, and narrower and convex at bottom region. In this study, as it is aimed to predict the kerf profile at a macro level, the roughness left behind the jet is neglected. The predicted width and the depth values are fitted on a Gaussian function as written below:

$h=h_{\max } \mathrm{e}^{\left(-\frac{x^{2}}{2 C^{2}}\right)}$, where $C=\frac{d}{4 \sqrt{2 \ln (2)}}$

In Eq. (17), $d$ is the kerf width, over which the water jet disperses after leaving the nozzle as shown in Fig. 7.
Therefore, a tapered kerf profile is generated having a taper angle of $\alpha$. Hashis and Du Plessis [20] reported that the dispersion angle may vary from 3 to $5^{\circ}$. Thus, the kerf width can be geometrically written as follows:

$d=2 x_{a} \tan (a / 2)+d_{0}$

where $x_{a}$ is standoff distance and $d_{0}$ is nozzle diameter.

Following the explanation of geometrical kerf profile prediction, in Section 3, the experimental verification is provided.

\section{Simulation of 3D workpiece geometry}

In this study, an approach for gradual update of the in-process workpiece (IPW) geometry throughout the AWJ process, is proposed. For such a purpose, the developed AWJ process model is integrated with a $3 \mathrm{D}$ workpiece representation scheme, which is based on signed distance field (SDF) methodology. The flow chart of the proposed approach is given in Fig. 8. In Step 1, the kerf profile is predicted using the proposed model in Section 2. Then, the revolved volume of the kerf profile is obtained in order to treat it as a soft cutting tool to integrate with the IPW volume, which is performed at Step 3. Finally, the soft tool volume together with the IPW volume is represented in the SDF scheme to perform the geometrical update.

\subsection{Signed distance field representation}

The signed distance field is a scalar function of space coordinates which gives the shortest distance to an object. The object is described by a domain $\Omega$ with the boundary $\partial \Omega$. The distance is most often measured by the Euclidian metric, here denoted by $\|\cdot\|$. The foot point $\overline{\mathrm{y}} \in \partial \Omega$, i.e. the orthogonal projection of a point $\mathrm{x} \in \mathrm{R} \wedge 3$ onto the object surface, is obtained as the minimizing argument.

$$
\bar{y}(x)=\arg \inf _{y \in \partial \Omega}\|x-y\|
$$

Fig. 6 Kerf profile calculation algorithm representation for the first three jet segments
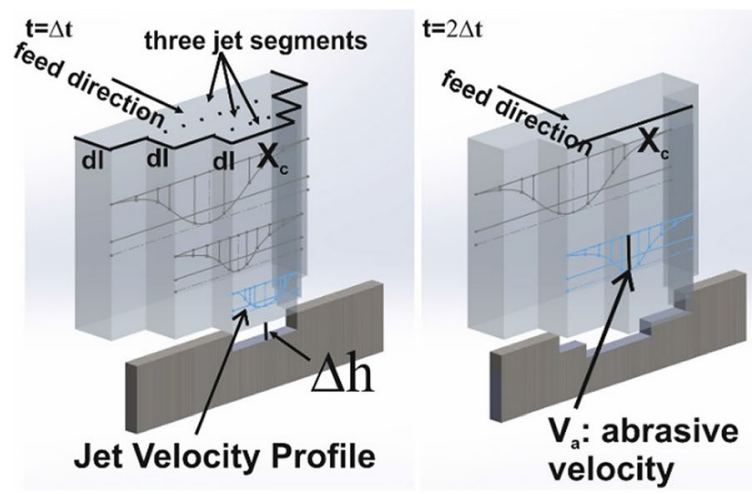


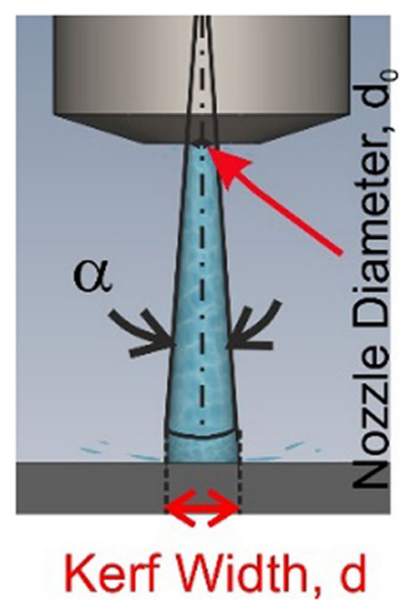

Fig. 7 Jet dispersion leading to kerf taper angle

The distance function then easily arises:

$d(\boldsymbol{x})=\|\boldsymbol{x}-\overline{\boldsymbol{y}}(\boldsymbol{x})\|$

Having the distance function at hand, the surface of the object can be represented as the zero-level set of this function:

$S=\left\{x \in \mathbb{R}^{3} \mid d(x)=0\right\}$

However, the distance function (19) does not carry information whether the point $x$ lies inside or outside of the object, which is handled by the signed distance function:

$d_{\mathrm{s}}(x)=\operatorname{sign}(x) d(x)$
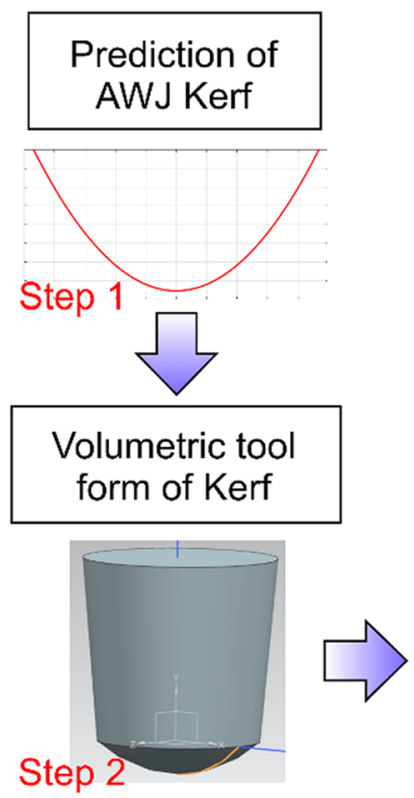

Fig. 8 3D IPW Simulation approach where the signum function is defined as follows:

$\operatorname{sign}(x)=\frac{[x-\bar{y}(x)] \cdot n}{\|[x-\bar{y}(x)] \cdot n\|}$

where $n$ is the unit outward normal vector of the object surface.

For simple shapes, the signed distance field (SDF) can be expressed analytically. However, for more complex geometries, SDF must be treated numerically. In practice, values of signed distance are sampled into discrete points near the object surface. The discrete points can be vertices of unstructured triangular [39] [40] or regular grids (i.e. voxel grid) [41]. More sophisticated approaches are based on parallel implementation of quadtrees and octrees [37, 42]. In the proposed approach, sparse block grid data structure is proposed [36]. It is a two-level data structure, which is depicted in Fig. 9, where the first rough level is covered by blocks that are either inside or outside the body. On the second level, the blocks through which the boundaries pass are sampled with higher resolution.

\subsection{Distance field update}

A specific feature of water jet simulation is the fact that not only the workpiece but also the water jet shape can change during machining. To consider the time variation of this "tool" shape, it was necessary to modify the distance function update procedure. For simplicity, we assume that the water jet shape/ tool is rotationally symmetrical, and its meridian section is described by piece-wise linear sections. Let us have a sampling point from the distance function grid, marked in red in Fig. 2. To update the distance field at this point, we need to know if the point is inside or outside the tool and what is its new distance from the surface. To this end, we first find the nearest point between the points that define the meridian section of the tool. In Fig. 9, this point is indicated in violet. We then project on two adjacent linear sections and choose the shorter one as the new distance. The sign is determined by the scalar product with the vector of the outer normal vector. In case, the sampling points lie within the dead-zone cones,

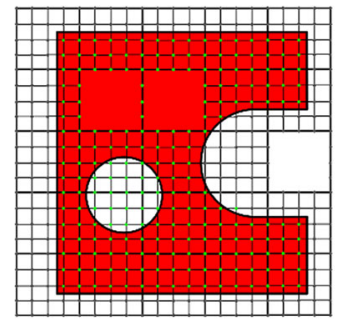

Step 3

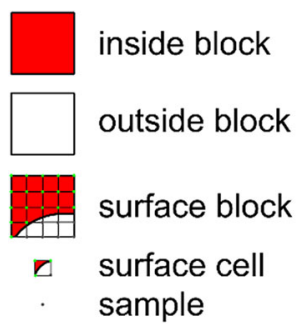

(a)

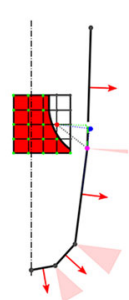

(b)
Fig. 9 Surface representation by SDF. (a) Sampling on a sparse block grid and (b) DF update using the waterjet plum envelope 
indicated by pink in Fig. 9, and then the closest point is the tool point itself.

\section{Experimental Verification}

In this section, experimental verification of the proposed AWJM process model and the IPW simulation framework is presented. First, the experimental setup is briefly explained, followed by the justification of Assumption 1 in Section 2.2 is provided through pure water jet machining experiments in Section 4.2. Then, the kerf prediction model is verified in Section 4.3 based on the experimental results existing in the literature and new machining tests performed in-house, as part of this study. The IPW simulation approach is verified in Section 4.4 by variable feed rate AWJM experiments.

\subsection{Experimental setup}

The experiments are performed on a 7-axis robotic AWJ milling system equipped with KUKA (C) 6-axis serial arm KR16Ultra $F$ robot over hanged on a linear gantry as shown in Fig. 10. The robot end effector is fitted with a $1.02-\mathrm{mm}$ diameter nozzle, where the water is pumped by KMT-Neoline (C) double intensifier pump. The tool path is generated by using the Siemens NX12 (C) CAM package (Fig. $10 \mathrm{a}$ and b) where the robot motion is verified and post-processed using the robotic machining module developed by Siemens NX12 (C) (Fig. 10c). As the controller has the KUKA.CNC function, the robot motion is provided by G-Codes. In the machining experiments, Si-based garnet abrasives were used having 80 mesh number, i.e. average diameter of 210 microns which are obtained from optical microscope as shown in Fig. 10 e and f. In all the experiments, the kerf profile is measured using NanoFocus uSurf Non-Contact 3D Profilometer.

\subsection{Pure WJM experiments}

Assumption 1 states that kinetic energy of water can be ignored with regard to the material removal, especially in AWJM of metallic materials. This is experimentally verified in this section by performing pure water jet machining (WJM) tests, i.e. without any abrasive particles, under four different conditions representing wide range of process parameters. Pressure levels of 150 and $350 \mathrm{MPa}$ are selected, where the feed rate levels were 1500 and $3000 \mathrm{~mm} / \mathrm{min}$ as listed in Table 1.

According to the results given in Table 1, the minimum kerf depth is obtained as 5 microns in experiment \#1, where the deepest kerf was obtained as 100 microns in experiment \#3, which is also repeated using abrasives as experiment\#3a in Table 1. The machined workpiece material and measured kerf profile after experiment 3 and experiment 4 are shown in Fig. 11 , as well

At the most energetic case, i.e. experiment \#3, the depth of the kerf is around 100 microns as shown in Fig. 11b, which is approximately $6 \%$ of the depth of 1624 microns, achieved by AWJM (See Table 1, experiment \#3a). In the other cases, the contribution of water energy in erosion is much less. For example, in experiment \#4, the kerf depth obtained by pure WJM is 18 microns (See Figure 11c), which $1.5 \%$ of the depth of 1133 microns, achieved by AWJM. Considering the very
Fig. 10 Experimental setup: (a, b) Typical tool path used in experiments, (c) Siemens NX() screen for robotic arm movement simulation, (d) robotic abrasive water jet configuration, (e) abrasives used in experiment, and (f) abrasive particle size distribution

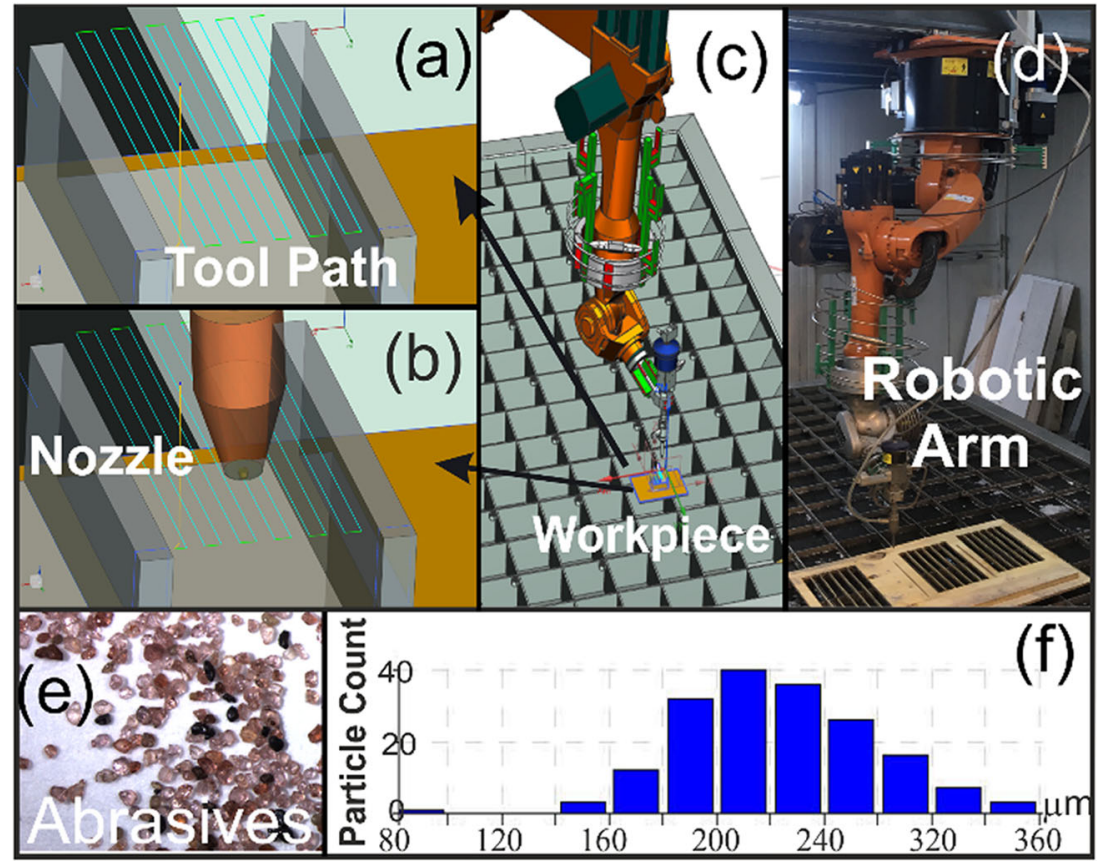


Table 1 Experimental conditions of pure WJM

\begin{tabular}{lllllll}
\hline Experiment & 1 & 2 & 3 & $3 \mathrm{a}$ & 4 & $4 \mathrm{a}$ \\
\hline Abrasive $(\mathrm{g} / \mathrm{s})$ & No & No & No & 2.4 & No & 2.4 \\
Pressure $(\mathrm{MPa})$ & 150 & 150 & 350 & 350 & 350 & 350 \\
Feed Rate $(\mathrm{mm} / \mathrm{min})$ & 1500 & 3000 & 1500 & 1500 & 3000 & 3000 \\
Kerf Depth $(\mu \mathrm{m})$ & 5 & 10 & 100 & 1624 & 18 & 1133 \\
\hline
\end{tabular}

low kerf depth achieved even at high pressure and relatively low feed rate, it can be said that contribution of pure WJM to material removal is negligible, and hence, Assumption 1 is experimentally justified.

\subsection{Verification of the AWJM process model}

Process model verification is performed in two phases, i.e. by comparison with literature data and in-house machining tests as discussed in this section. Comparison with literature data involves 17 tests and in-house machining tests involve 8 cutting tests.

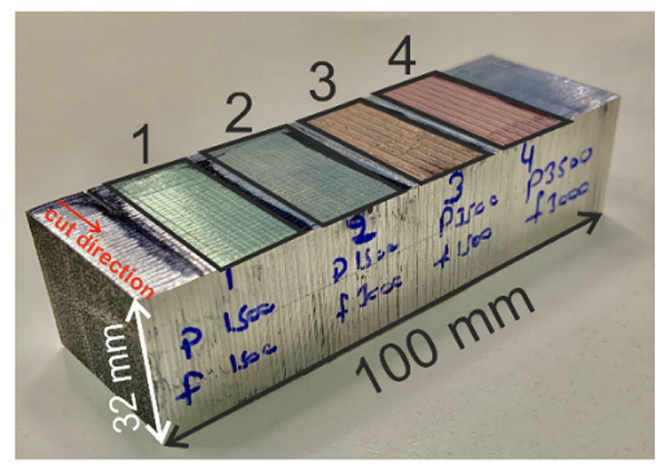

(a)

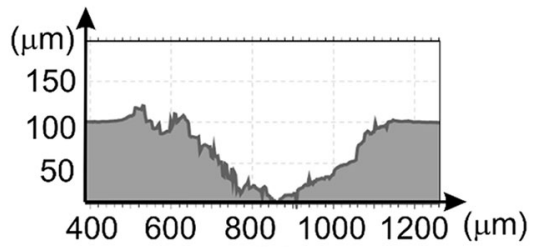

(b)

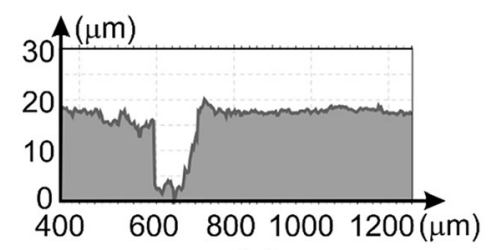

(c)

Fig. 11 Experimental Results for pure WJM. (a) Workpiece material used in pure WJM, (b) kerf profile after experiment \#3, (c) Kerf profile after experiment \#4

\subsubsection{Comparison with the literature}

In the literature, there are several AWJM cutting tests, whose kerf depth information is available and performed on various materials. In this regard, comparison with such already existing data is considered a reasonable way of verifying the proposed model in this study. The details of the literature data used in this section are given in Table 2. In all the cases, the stand-off distance (SOD) was set to $3 \mathrm{~mm}$. The cases from 1 to 12 were taken from the study of Pal and Choudhury [43], the cases from 13 to 16 were taken from Axinte at al. [29], and case 17 was taken from Fowler et al. [10]. The kerf depth comparisons are presented in Fig. 12a, whereas the error analysis is presented in Fig. 12b.

In Fig. $12 \mathrm{a}$, it is seen that the proposed model provides a good match with the experimental data existing in the literature, in terms of both kerf depth and the trend in the variation. For instance, at high jet traverse speeds, the kerf depth is low and at lower jet traverse speeds, the kerf depth increases. The error analysis shows that 13 out of 17 cases are in an error margin of $20 \%$, whereas 15 of 17 cases are in $30 \%$ of error margin. Such a reasonable agreement with the literature data demonstrates the validity and generality of the proposed model, which shows that even without a calibration test, by knowing the process parameters and material type, the proposed model can predict the kerf depth at a reasonable accuracy. Considering that the literature data involves experiments performed on various materials such as AL6061, SS301, Ti6AL4V, and $\mathrm{SiC}$, it can be said that the proposed model in this study is also verified on several materials. Together with the elimination of any prior calibration tests, this study proposes a generalized AWJM process model as a major contribution to the literature.

\subsubsection{In-house experiments}

Considering that the aim of the experiments was to verify the kerf profile predictions and low cost of aluminium alloys, AL6061-T6 is used in the in-house verification experiments. However, it is noteworthy to state that AL6061-T6 is an easyto-cut material and with no challenges in high-speed milling. The experimental conditions were selected within the ranges commonly used for AWJM processes in the literature, as listed in Table 3 . The test parameters were selected as feed rate, pressure, standoff distance, and abrasive flow rate. The orifice size, nozzle length, mesh size, and nozzle diameter effects are verified by the data existing in the literature. The size of abrasives was \#80 and nozzle length and diameter were $90 \mathrm{~mm}$ and $0.762 \mathrm{~mm}$, respectively, whereas the orifice diameter was $0.254 \mathrm{~mm}$.

In the tests, to check uniform process conditions, 5 cutting steps were used on each sample. The tool path used in the cutting tests is illustrated in Fig. $10 \mathrm{a}$ and b. Considering the 
Table 2 Experimental dataset extracted from the literature

\begin{tabular}{|c|c|c|c|c|c|c|c|c|}
\hline Case & Material & $\begin{array}{l}\text { Mesh } \\
\#\end{array}$ & $\begin{array}{l}\text { Pressure } \\
(\mathrm{MPa})\end{array}$ & $\begin{array}{l}\text { Feed } \\
(\mathrm{m} / \mathrm{min})\end{array}$ & $\begin{array}{l}\text { Abrasive } \\
\text { rate }(\mathrm{g} / \mathrm{s})\end{array}$ & $\begin{array}{l}\text { Nozzle } \\
\text { diameter } \\
(\mathrm{mm})\end{array}$ & $\begin{array}{l}\text { Orifice } \\
\text { diameter } \\
(\mu \mathrm{m})\end{array}$ & $\begin{array}{l}\text { Nozzle } \\
\text { length } \\
(\mathrm{mm})\end{array}$ \\
\hline $1^{*}$ & AL6061 & 80 & 172 & 4.5 & 3.76 & 0.762 & 330 & 101.6 \\
\hline $2^{*}$ & AL6061 & 80 & 241 & 4.5 & 3.76 & 0.762 & 330 & 101.6 \\
\hline $3 *$ & AL6061 & 120 & 172 & 4.5 & 3.76 & 0.762 & 330 & 101.6 \\
\hline $4^{*}$ & AL6061 & 120 & 241 & 4.5 & 3.76 & 0.762 & 330 & 101.6 \\
\hline $5^{*}$ & SS301 & 80 & 172 & 4.5 & 3.76 & 0.762 & 330 & 101.6 \\
\hline $6^{*}$ & SS301 & 80 & 241 & 4.5 & 3.76 & 0.762 & 330 & 101.6 \\
\hline $7 *$ & SS301 & 120 & 172 & 4.5 & 3.76 & 0.762 & 330 & 101.6 \\
\hline $8^{*}$ & SS301 & 120 & 241 & 4.5 & 3.76 & 0.762 & 330 & 101.6 \\
\hline $9^{*}$ & Ti6Al4V & 80 & 172 & 4.5 & 3.76 & 0.762 & 330 & 101.6 \\
\hline $10^{*}$ & Ti6Al4V & 80 & 241 & 4.5 & 3.76 & 0.762 & 330 & 101.6 \\
\hline $11^{*}$ & Ti6Al4V & 120 & 172 & 4.5 & 3.76 & 0.762 & 330 & 101.6 \\
\hline $12^{*}$ & Ti6Al4V & 120 & 241 & 4.5 & 3.76 & 0.762 & 330 & 101.6 \\
\hline $13 * *$ & $\mathrm{SiC}$ & 80 & 345 & 1.7 & 11.76 & 1 & 254 & $\mathrm{~N} / \mathrm{A} * * * *$ \\
\hline $14 * *$ & $\mathrm{SiC}$ & 80 & 345 & 1.3 & 11.76 & 1 & 254 & \\
\hline $15^{* *}$ & $\mathrm{SiC}$ & 80 & 345 & 0.9 & 11.76 & 1 & 254 & \\
\hline $16^{* *}$ & $\mathrm{SiC}$ & 80 & 345 & 0.5 & 11.76 & 1 & 254 & \\
\hline $17 * * *$ & Ti6A14V & 80 & 138 & 0.2 & 5 & 1 & 250 & 75 \\
\hline
\end{tabular}

*Pal and Choudhury [43]

**Axinte et al. [29]

***Fowler et al. [10]

$* * * *(25 \mathrm{~mm}$ used in the simulations as observed from the experimental setup) inherent geometrical inaccuracy of the controlled depth AWJM processes, the kerf profile was measured at several sections along the jet traverse direction, using Nano Focus uSurf (c) Non-Contact 3D Profilometer. Representative 3D measurements of the kerf geometries are given in Fig. 13, where the kerf depth, width, and measurement section are also illustrated. The kerf depth and width are measured by taking a cross section perpendicular to the jet traverse direction.

The comparison of the predicted kerf depth with the experimental values is given in Fig. 14. It is seen that in most of the cases, a good agreement is observed between simulations and experiments in terms of both trend and kerf depth. In only one of the cases, the error margin is $30 \%$ and the rest is less than $25 \%$. The average error is around $15 \%$. The maximum error is observed when the abrasive rate is relative higher, which may be attributed to the inaccurate supply of the abrasive in the AWJM system.

Comparison of kerf depth is not the only measure in verification of the proposed model. In this regard, the width, depth, and profile of the kerf are compared in Fig. 15. The individual comparison of kerf profiles obtained in 8 cutting experiments shows that in all the tests, the kerf width is predicted with almost no error, where the kerf depth prediction errors, previously mentioned in Fig. 14b, are visually
Table 3 Experimental parameter values and results

\begin{tabular}{ccccc}
\hline Experiment & Pressure $(\mathrm{MPa})$ & Feed (mm/min) & SOD (mm) & Abrasive flow rate $(\mathrm{g} / \mathrm{s})$ \\
\hline 1 & 150 & 1500 & 2 & 1 \\
2 & 350 & 2000 & 3 & 1 \\
3 & 250 & 2000 & 2 & 6 \\
4 & 350 & 3000 & 2 & 10 \\
5 & 350 & 1000 & 5 & 2.4 \\
6 & 350 & 1500 & 5 & 2.4 \\
7 & 350 & 3000 & 5 & 2.4 \\
8 & 300 & 3000 & 5 & 2.4 \\
\hline
\end{tabular}


Fig. 12 Comparison of the proposed model with literature data. (a) Kerf depth comparison and (b) error distribution

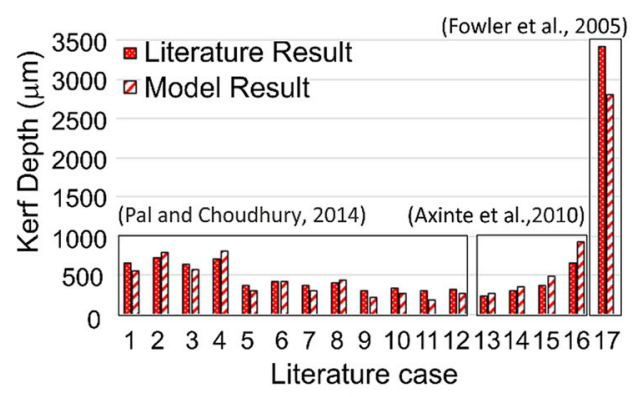

(a)

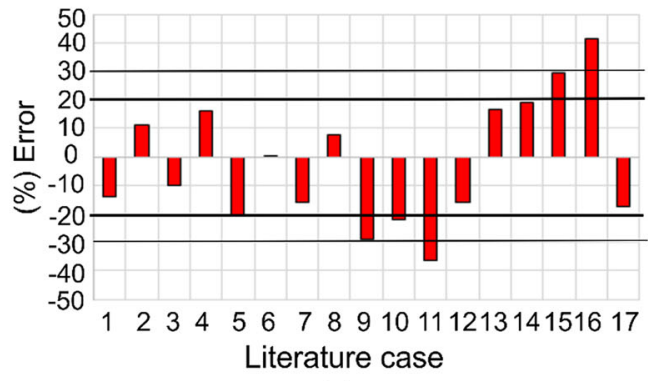

(b) illustrated, so that the effect of kerf depth errors on the resulting kerf profile is visually understood.

Considering the results in the literature and the in-house experiments given in this study, the below trend analysis can be deducted with regard to the effect of process parameters on the process outputs.

- The kerf depth increases with decreasing feed rate and increasing water pressure.

- Abrasive flow rate has significant effect on material removal and hence kerf depth. Once the abrasive flow rate is increased even at relatively high jet traverse speeds, deep kerf geometries can be achieved.

- According to the water pressure, orifice diameter, and abrasive size, choking may be observed after a level of abrasive flow rate.

\subsubsection{Error analysis}

In the experimental verification, results of both literature and in-house experiments are presented. There were some cases demonstrating kerf depth error more than $20 \%$, which may be quite far from providing a reasonable prediction. The major reasons for the errors may be averaging in measurements, inaccuracies related to the process itself, non-uniformities in water and abrasive flow rates, nonhomogeneous property of the abrasive particles in terms of composition and size, partial chocking of the abrasives, and inaccurate reading of the water flow rate from the pump manufacturer. To address potential
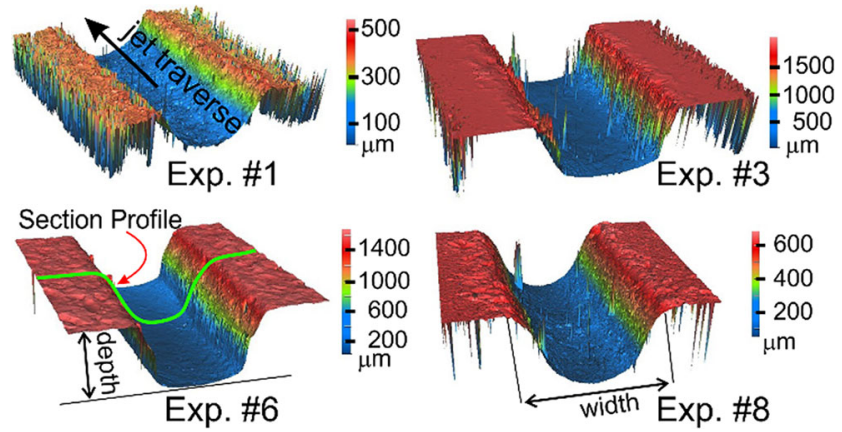

Fig. 13 Representative of 3D kerf measurements reasons of such a discrepancy, a comprehensive error analysis is provided in this section.

As the predictions of the proposed model and results in the literature are compared, it is observed that at lower pressure values, the proposed model tends to predict the kerf depth lower than the values given in the literature. However, at higher pressure values, the model tends to predict the kerf width higher than the literature result. As the literature data 14,15 , and 16 are observed, it can be said that the error ratio increases at relatively much lower feed rate values, which is a common issue in AWJM modelling in the literature. However, the maximum error ratio is around $20 \%$, which is still reasonable for prediction purposes. Another reason for errors would be the fluctuations on the abrasive flow rate throughout the AWJM cut. Nonetheless, it can be concluded that the proposed model can predict the kerf depth accurate enough for planning of roughing passes using controlled depth AWJM approach.

\subsection{Simulation of in process workpiece (IPW)}

In this section, the IPW simulation approach is verified by two machining experiments, where the jet traverse speed is varied based on two ruling functions (see Fig. 16a) to create variable kerf profile along the jet traverse direction considering the significant effect of jet traverse speed on kerf depth. The kerf profile is predicted using the corresponding jet traverse speed at each tool path point, which is then used to update the softtool profile in the SDF based workpiece-jet plum representation scheme.

In the cutting experiments, $200 \mathrm{MPa}$ pressure, $2.4 \mathrm{gr} / \mathrm{s}$ of abrasive flow are used. In the first cut, jet traverse speed is varied linearly, whereas in the second cut, sinusoidal variation is implemented. In the linear variation, as the jet traverse speed approaches to the peak value, near the middle portion of the tool path, the kerf depth is expected to be less, thereafter the kerf depth increases as the jet traverses towards the end of the path. Similarly, in the sinusoidal variation, the kerf depth is expected to vary sinusoidally with increased and decreased kerf depth at lower and higher traverse speed, respectively.

The workpiece is sectioned along the jet traverse speed direction to obtain the cross-sectional view for through 
Fig. 14 Experimental verification of the kerf depth predictions. (a) Kerf depth comparison and (b) error distribution

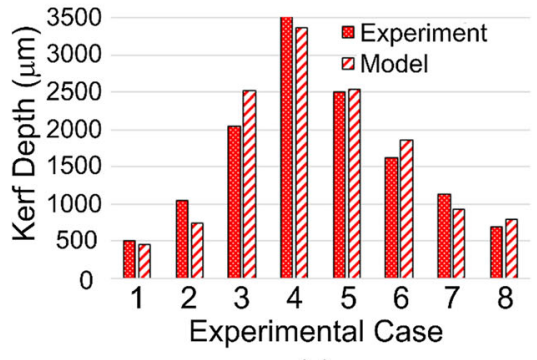

(a)

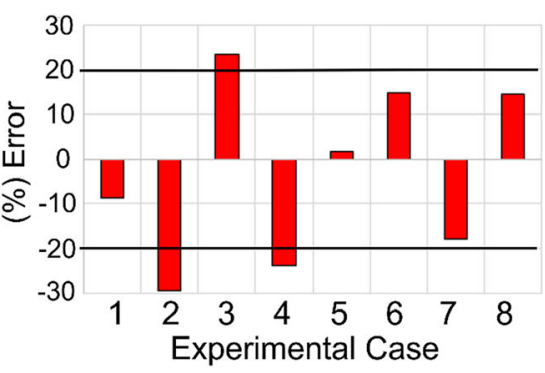

(b) comparison with simulations. The $3 \mathrm{D}$ view of the obtained cross sections from the physical part and simulated IPW are shown in Fig. 16c, which are put on top of each other in Fig. $16 \mathrm{~d}$. The cross-sectional comparison of the experiment and simulation of the IPW demonstrates a reasonable agreement as highlighted in Fig. 16d. However, still there is some amount of undulated overcut and undercut in the physical part (see Fig. 16d), which is most probably due to the unstable abrasive supply throughout the jet traverse. Considering the good agreement in predicted profiles, it can be said that the proposed volumetric simulation scheme is accurate enough for planning of rough AWJM passes in controlled depth cutting.

\section{Conclusions}

In this study, a novel analytical model is proposed for prediction of the kerf shape in AWJM processes, which is then used for prediction of the IPW geometry in controlled depth AWJM. The IPW geometry and the volume of the jet plum are represented in a signed distance field-based scheme.

The proposed AWJM process model relies on process parameters such as pump pressure, abrasive flow rate, feed rate, standoff distance, nozzle size, abrasive size, orifice size, and the machinability number of the workpiece material. The energy conservation is used to predict kerf depth. By knowing the pump pressure, firstly the water jet velocity is obtained by Bernoulli Equation considering compressibility effect on the orifice. With the help of conservation of momentum, the velocity of the abrasive in the nozzle is calculated. Velocity is predicted by taking water jet momentum. After finding velocity of the particle, it is converted to kinetic energy by calculating the mass of the abrasive. The kinetic energy is equated to the specific cutting energy of the workpiece material, which is related to the machinability number as available in the literature. By doing so, the requirement for any type of calibration tests is eliminated.

Since the abrasive velocity varies along radial direction of exposure area, the jet cross section is divided into small segments, where the segmental velocity is calculated by considering velocity profile. Each segment causes material removal, which results in kerf geometry. The predictions are compared with the data existing in the literature and in-house performed experiments, where good agreement was observed in most of the cases. The maximum error on kerf depth prediction was observed around $30 \%$, where mostly the error was less than $20 \%$ with an average error rate of $15 \%$.

In the literature, there are models to predict kerf profile in AWJM; the presented model proposes a novel approach in
Fig. 15 Comparison of kerf profiles
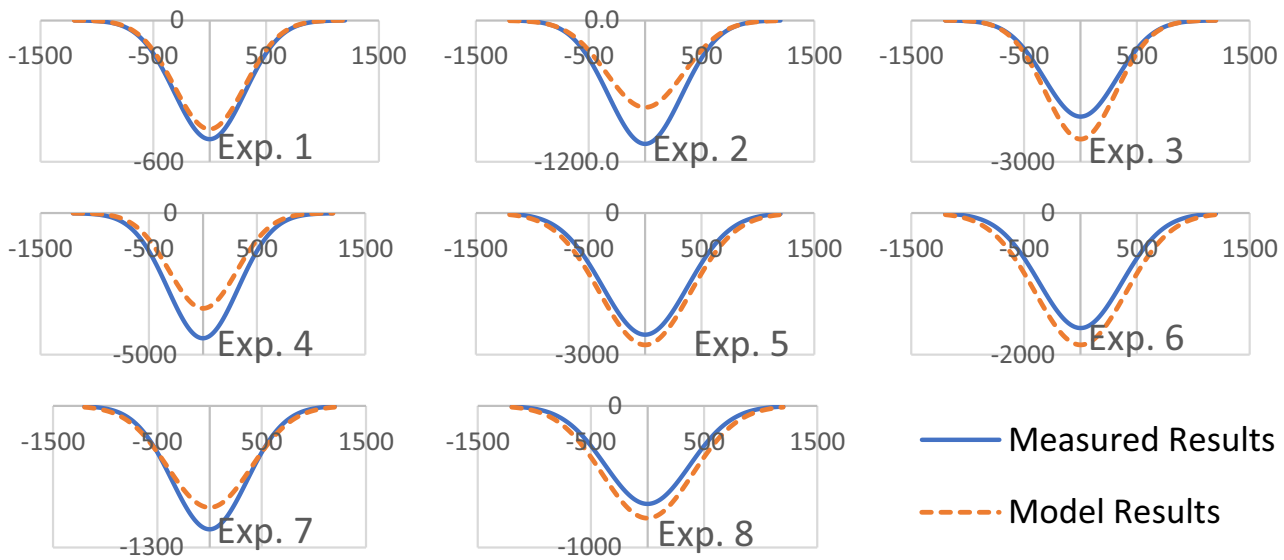

Measured Results - Model Results 
Fig. 16 IPW update verification experiments. (a) Traverse speed profile, (b) workpiece used in the experiments, (c) 3D sectional view of the part, (d) Cross section comparison

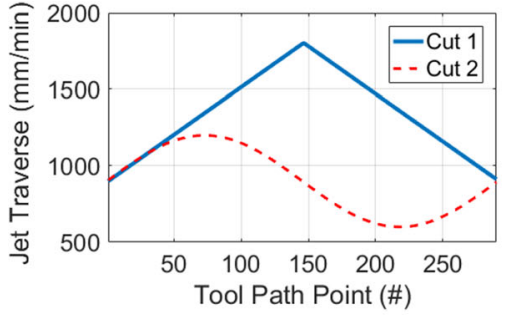

(a)

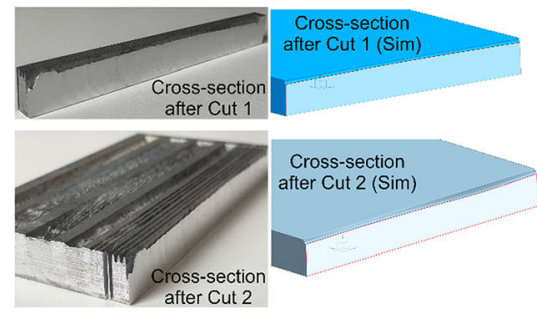

(c)

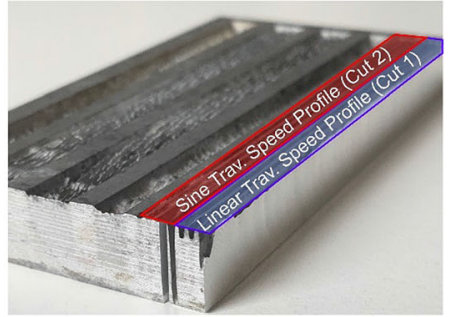

(b)

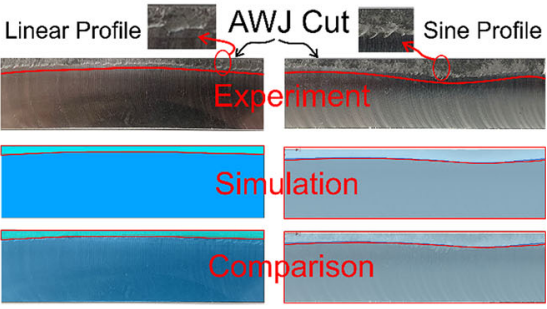

(d) terms of independence of prior calibration experiments. With the help of this method, the kerf profile can be predicted by knowing the process parameters and the workpiece material. There is no need to identify any experimental constant, which significantly increases the generality of AWJM process modelling. In addition, as the proposed model is analytical, it provides significantly fast simulation compared to FEMbased models.

Simulation of IPW geometry in controlled depth AWJM is performed by interfacing the kerf prediction model with a signed distance field representation scheme. The kerf profile is analytically predicted under varying AWJM conditions, which is then implemented as a soft cutting tool in the form of a jet plum in order to update the in process workpiece (IPW) geometry. The IPW prediction approach is verified with variable feed rate AWJM cutting tests, where the predicted profile of the workpiece along the jet traverse direction, i.e. cross section, is simulated in a good agreement with the experimental results. In general, it can be said that one of the major error sources is the inaccurate abrasive supply in the system, which causes undulation in the generated kerf.

\section{Code availability Not applicable.}

Author contribution All authors contributed in the process of critical literature review. All author contributed in writing and revising the manuscript. Specifically, Yigit Ozcan contributed to the development of kerf geometry prediction model. Taner Tunc contributed to integration of the kerf geometry model with the 3D workpiece simulation model, experimental studies. Beril Cetin contributed to the experimental studies. Jan Kopacka contributed to preparation of the 3D workpiece simulation model for AWJM simulation. Matej Sulitka contributed to the evaluation of the simulation and experimental results.

Funding This study is funded by The Scientific and Technological Research Council or Turkey (TUBITAK) under grant 217M704.
Data availability Not applicable

\section{Declarations}

Ethical approval The manuscript in part or in full has not been submitted or published anywhere. The manuscript will not be submitted elsewhere until the editorial process is completed.

Consent to participate Not applicable.

Consent to publish The author transfers to Springer the non-exclusive publication rights.

Competing interests The authors declare no competing interests.

\section{References}

1. Konig W, Cronjager L, Spur G, H-K T, Vigneau M, Zdeblick WJ (1991) Machining of new materials. Process. Adv. Mater. 1:11-26

2. Lauwers B, Klocke F, Klink A, Tekkaya AE, Neugebauer R, Mcintosh D (2014) Hybrid processes in manufacturing. CIRP Annals 63(2):561-583

3. Kong MC, Srinivasu D, Axinte D, Voice W, McGourlay J, Hon B (2013) On geometrical accuracy and integrity of surfaces in multimode abrasive waterjet machining of NiTi shape memory alloys. CIRP Annals. 62(1):555-558

4. Axinte DA, Karpuschewski B, Kong MC, Beaucamp AT, Anwar S, Miller D, Petzel M (2014) High energy fluid jet machining (HEFJet-Mach): from scientific and technological advances to niche industrial applications. CIRP Annals 63(2):751-771

5. Liao Z, Sanchez I, Xu D, Axinte D, Augustinavicius G, Wretland A (2020) Dual-processing by abrasive waterjet machining-a method for machining and surface modification of nickel-based superalloy. Journal of Materials Processing Technology 285:116768

6. Matsumura T, Muramatsu T, Fueki S (2011) Abrasive water jet machining of glass with stagnation effect. CIRP Annals 60(1): 355-358 
7. Siores E, Wong WCK, Chen L, Wager JG (1996) Enhancing abrasive waterjet cutting of ceramics by head oscillation techniques. CIRP Annals 45(1):327-330

8. Wei H, Peng C, Gao H, Wang X, Wang X (2019) On establishment and validation of a new predictive model for material removal in abrasive flow machining. International Journal of Machine Tools and Manufacture 138:66-79

9. Momber, A. W., \& Kovacevic, R. (2012). Principles of abrasive water jet machining. Springer Science \& Business Media.

10. Fowler G, Shipway P, Pashby I (2005) Abrasive water-jet controlled depth milling of Ti6A14V alloy-an investigation of the role of jet-workpiece traverse speed and abrasive grit size on the characteristics of the milled material. Journal of Materials Processing Technology. 161:407-414

11. Azmir MA, Ahsan AK (2009) A study of abrasive water jet machining process on glass/epoxy composite laminate. Journal of Materials Processing Technology 209(20):6168-6173

12. Löschner P, Jarosz K, Niesłony P (2016) Investigation of the effect of cutting speed on surface quality in abrasive water jet cutting of 316L stainless steel. Procedia Engineering 149:276-282

13. Shukla R, Singh D (2017) Experimentation investigation of abrasive water jet machining parameters using Taguchi and Evolutionary optimization techniques. Swarm and Evolutionary Computation 32:167-183

14. Uthayakumar M, Khan MA, Kumaran ST, Slota A, Zajac J (2016) Machinability of nickel-based superalloy by abrasive water jet machining. Materials and Manufacturing Processes 31(13):1733-1739

15. Wang S, Zhang S, Wu Y, Yang F (2017) Exploring kerf cut by abrasive waterjet. The International Journal of Advanced Manufacturing Technology 93(5-8):2013-2020

16. Yuvaraj N, Kumar MP (2017) Investigation of process parameters influence in abrasive water jet cutting of D2 steel. Materials and Manufacturing Processes 32(2):151-161

17. Finnie I (1960) Erosion of surfaces by solid particles. Wear. 3:87103

18. Bitter JJW (1963) A study of erosion phenomena: Part I. Wear. 6:521

19. Bitter JGA (1963) A study of erosion phenomena: Part II. Wear 6(3):169-190

20. Hashish M, Du Plessis M (1979) Prediction equations relating high velocity jet cutting performance to stand off distance and multipasses. Journal of Engineering for industry 101:311-318

21. Tazibt A, Parsy F, Abriak N (1996) Theoretical analysis of the particle acceleration process in abrasive water jet cutting. Computational Materials Science 5:243-254

22. Capello E, Groppetti R (1992) On an energetic semi-empirical model of hydro-abrasive jet material removal mechanism for control and optimization. In: Jet cutting technology. Springer, Dordrecht, pp 101-120

23. Raju SP, Ramulu M (1994) Predicting hydro-abrasive erosive wear during abrasive waterjet cutting: Part II-experimental study and model verification. ASME-PUBLICATIONS-PED 68:381-381

24. Momber AW, Kovacevic R (1999) An energy balance of highspeed abrasive water jet erosion. Proceedings of the Institution of Mechanical Engineers, Part J: Journal of Engineering Tribology 213(6):463-472

25. Hoogstrate AM, Karpuschewski B, Van Luttervelt CA, Kals HJJ (2002) Modelling of high velocity, loose abrasive machining processes. CIRP Annals. 51(1):263-266

26. EITobgy M, Ng EG, Elbestawi MA (2005) Modelling of abrasive waterjet machining: a new approach. CIRP Annals. 54(1):285-288
27. Pi VN, Tuan NQ (2009) Necessary cutting energy in abrasive waterjet machining. In: Advanced Materials Research, vol 76. Trans Tech Publications Ltd, pp 351-356

28. Ahmed DH, Naser J, Deam RT (2016) Particles impact characteristics on cutting surface during the abrasive water jet machining: numerical study. Journal of materials processing technology 232 : $116-130$

29. Axinte DA, Srinivasu DS, Billingham J, Cooper M (2010) Geometrical modelling of abrasive waterjet footprints: a study for 90 jet impact angle. CIRP Annals 59(1):341-346

30. Billingham J, Miron CB, Axinte DA, Kong MC (2013) Mathematical modelling of abrasive waterjet footprints for arbitrarily moving jets: Part II-Overlapped single and multiple straight paths. International Journal of Machine Tools and Manufacture 68:30-39

31. Gilles P, Sultan T, Cohen G, Rubio W (2017) A new cutting depth model with rapid calibration in abrasive water jet machining of titanium alloy. The International Journal of Advanced Manufacturing Technology 93(5-8):1499-1512

32. Anwar S, Axinte DA, Becker AA (2013) Finite element modelling of overlapping abrasive waterjet milled footprints. Wear 303(1-2): 426-436

33. Kumar N, Shukla M (2012) Finite element analysis of multi-particle impact on erosion in abrasive water jet machining of titanium alloy. Journal of Computational and Applied Mathematics 236(18):4600 4610

34. Altintas Y, Kersting P, Biermann D, Budak E (2014) Virtual process systems for part machining operations. CIRP Annals 63(2): 585-605

35. Jones MW, Bærentzen JA, Sramek M (2006) 3D distance fields: a survey of techniques and applications. IEEE Trans. Visual. Computer Graphics. 12:581-599

36. Jamriška O., Havran V. (2010) Interactive ray tracing of distance fields, in: Proc. 14th Cent. Eur. Semin. Comput. Graph., Wien.

37. Sullivan A, Erdim H, Perry RN, Frisken SF (2012) High accuracy NC milling simulation using composite adaptively sampled distance fields. Computer. Aided Des. 44:522-536

38. Hashish M (2013) Waterjet machining process. Handbook of Manufacturing Engineering Technology:1-30

39. Xu M, Thompson PM, Toga AW (2004) An adaptive level set segmentation on a triangulated mesh. IEEE Transactions on Medical imaging 23(2):191-201

40. Wu Y, Man J, Xie Z (2014) A double layer method for constructing signed distance fields from triangle meshes. Graphical models 76(4):214-223

41. Chang, B., Cha, D., \& Ihm, I. (2008). Computing local signed distance fields for large polygonal models. In Computer Graphics Forum (Vol. 27, 3, pp. 799-806). Oxford, UK: Blackwell Publishing Ltd.

42. Yu H, Xie J, Ma KL, Kolla H, Chen JH (2015) Scalable parallel distance field construction for large-scale applications. IEEE transactions on visualization and computer graphics 21(10):1187-1200

43. Pal VK, Choudhury SK (2014) Fabrication and analysis of micropillars by abrasive water jet machining. Procedia materials science 6:61-71

Publisher's note Springer Nature remains neutral with regard to jurisdictional claims in published maps and institutional affiliations. 